\title{
Situation Analysis of Health Club in Pingxiang City
}

\author{
Liling-Zeng \\ Pingxiang College of physical education \\ Pingxiang, Jiangxi, 337055
}

\begin{abstract}
In this paper, through literature, materials, testing and other research methods, the current status of the fitness industry in Pingxiang as the object of investigation, explore the current development of fitness Sporting Club Hotel in Pingxiang. With the improvement of people's living standard, the sports fitness club industry in Pingxiang has made great progress. To explore the current situation of the development of fitness clubs in Pingxiang, it is found that the marketing publicity mode is backward, the coaches' professional quality is low, the staff team service consciousness needs to be improved, and the related policies are not perfect. And the corresponding countermeasures are put forward to promote the sustained, healthy and rapid development of Pingxiang fitness industry. To provide practical guidance for the fitness industry in Pingxiang.
\end{abstract}

Keywords-Pingxiang; Fitness club; Status quo; Countermeasures

\section{INTRODUCTION}

China's economy continues to adhere to many years of high growth, people's living standards have improved significantly, material life has been met, prompting more and more people pay more attention to health. The slogan of "exercise for an hour a day, live well for 50 years, and live a happy and healthy life" makes people realize the importance of healthy life and begin to exercise healthily. However, because the pace of urban life is very fast, and a lot of people living environment, constitute a few occasions to provide fitness, the park has become a common flow of residents occasions, but the park can expand the fitness activities are very few. The lack of fitness needs provided opportunities for the fitness industry, however, the fitness club in the development process also presents some difficulties, this research has pointed out the research of fitness club visits, to analyze the existing problems, promote the fitness industry toward a more scientific direction to development [1].

\section{PINGXIANG FITNESS CLUB ANALYSIS OF THE STATUS} QUO

\section{A. Distribution and internal situation of fitness clubs in Pingxiang}

Through on-the-spot investigation and access to relevant personnel, to understand the Pingxiang City Fitness Club in the Emerald City V3 International Fitness Club, fitness club, Century Square resol Hakodate, sports center Parco Corrientes fitness club, fitness, Beiqiao 100 Run Da mall 36 V3 International Fitness Club, Runda mall 17 floor of the Bentley fitness club. Each gym has the same equipment, many including treadmills, fitness equipment, dynamic bikes, etc., but the difference is that fitness clubs have their own characteristics, such as: run up to V3 International Fitness Club in the air pool. In general, the differences in the fitness environment and fitness equipment in the gym determine the level of the difference, and thus cause the price difference.

\section{B. Pingxiang fitness club coach situation}

There are several gym coaches in Pingxiang, which are basically divided into private coaches and group calisthenics coaches. Only "V3 International Fitness" and "fitness" have professional swimming coaches. The aerobics coach as an important band aerobics, belly dance, yoga and other groups of courses, other dance class gymnastics coach basic from various places invited coach, has been learning about the project and dance classes. And private education as a member of the special one to one direction, each section of private teaching fees ranged from 80 to 160 yuan

\section{Membership of fitness clubs in Pingxiang}

Because there are differences in the fitness club in Pingxiang specifications and grades, absolutely speaking, the middle and high-end fitness club membership structure and the low-end fitness club member structure is different. The reason for these conditions is that the difference of the price of the fitness card is due to the difference of the hardware equipment of the club, so that the different consumer groups are separated. In the low end of the gym, one year card price is probably between 480 - 880 yuan, and the high-end fitness club's annual card is between 1600 - 2500 yuan. Generally speaking, the main cause of the difference in the structure of the population is the difference in consumption.

\section{Pingxiang City Sports Health Club related laws and regulations to be improved}

With the economic development in our country, the progress of people's living and the continuous expansion of the sports industry, people's awareness of sports and fitness has also been continuously improved. The number of people participating in sports and fitness has been added. At the same time, some questions have also appeared [2], for example: Interspersed, unknown responsibilities of the food industry, the industry standards, such as the cost of improper Changsha and the existence of it affects the interests of operators and consumers. Government lax regulation, ticket prices are too high, has affected the consumption of low-middle-income people into the form of physical fitness club sports spending enthusiasm. In particular, the impact of the price has always affected the health club's profits. If the government does not have time to work with businesses, taxation, layout, price and 
other departments together to develop fitness clubs related laws and regulations for the fitness club to create a good external environment, will certainly lead to some health clubs, especially those large-scale investment, large-scale The club faces great economic losses or even the risk of shutting down. In recent years, with the deepening of the establishment of the legal system, have successively presented the policies and regulations on the sports market. Pingxiang City has not yet formulated a relevant laws and regulations to bind and standard sports market, which led to a series of questions. The rights of consumers and managers can not be well protected.

\section{STUDY ON THE DEVELOPMENT COUNTERMEASURES OF FITNESS CLUBS IN PINGXIANG}

\section{A. Pingxiang fitness club marketing mode needs to be improved}

At present, the important marketing form adopted by Pingxiang fitness club is business promotion. The main ways of business promotion are discount, gift card, gift, experience card, lottery and so on. Advertising is a symbol of strong, but advertising mode is to issue leaflets, $92 \%$ of companies adopt leaflets propaganda way, the efficiency is very low. Personnel promotion and public relations marketing is important to give some help, as well as membership telephone publicity and promotion [3], so constitute publicity model limitations. According to a statistical test of the Internet, people in the fitness club fitness in $92 \%$, they will check the necessary information, therefore, fitness clubs in the media should be a concrete analysis of the current members and has potential members of the network, television, magazines and other cognitive level before. But in terms of the current situation of Pingxiang fitness club, there are few fitness clubs with their own websites. Therefore, the fitness club in Pingxiang should use all kinds of media to expand its popularity and reputation.

\section{B. The professional quality of private coaches needs to be improved}

There are many coaches in the fitness club in Pingxiang. Such as dance coaches, yoga coaches and so on, these coaches important work is to teach fitness skills, while leading everyone to train together. Private coaches do not only help members to improve their body shape, but also to monitor the change of the members' body target. Private coaches should have rich knowledge of sports, nutrition and psychology, and can realize the personal trainer service well. The survey found that, at present, the private coaches in Pingxiang are part of the undergraduate sports college graduates, sports common sense can be standardized. But $95 \%$ of the Personal Trainer Certificate and community sports instructor certificate is obtained through short-term training, fitness knowledge of personal trainer is too little. At the same time, $95 \%$ of coaches do not have medical or fitness medicine common sense, $90 \%$ of coaches do not have true nutrition knowledge training, very few coaches have nutritionist qualification, comprehensive data shows that the current private coaches in Pingxiang business quality is low.

\section{The overall quality of Pingxiang fitness club service team needs to be improved}

A club team will decide the future service quality longterm development organization structure to improve the quality of service to enhance competitiveness plays an important role as the organizational structure of clear division of responsibilities between departments to contact and communication, interaction, division and cooperation, and strive towards the same goal. Effect of organizational structure is affected by many elements of the club, fitness club difference found in the sampling test, the common organization of fitness clubs in Pingxiang city is not perfect, the management and operation of the important (Sales Department), mainly by the staff of coaches, site protection treatment personnel, sales personnel.

\section{The municipal government may establish the corresponding policy standard fitness club}

The municipal government can establish and adopt some corresponding laws, regulations and policies to standardize the fitness club, and successfully develop a good environment for it. The municipal government may, in conjunction with the relevant departments, take into account the formation of the layout of commercial sports clubs, promulgating corresponding regulations and policies, Quantity control, reasonable layout, constitute a network grade commercial sports fitness club organization to meet the economic strength of the middle-aged and young sports spending groups fitness needs. In setting up a commercial sports and fitness club, the government can adopt policies and support methods. Expanding the demand for sports, fitness, and entertainment industry the government and all sectors of society support and help the government to give some preferential policies to stimulate the enthusiasm of investors and managers and invent a good environment for their smooth development.

\section{FITNESS CLUB EXPERIENCE MARKETING STRATEGY OF THE BASIC REALIZATION OF THE PATH}

\section{A. Experience the theme design}

Experience themed design is fitness club managers and marketing team based on the fitness consumer demand for a reasonable classification, closely around an experience theme, all related services carefully designed, and strictly implemented. Health club rent, personnel, equipment costs are basically fixed, it is difficult to compress, profit model is relatively simple, fitness consumers also want to enjoy more and more inexpensive service. Through the theme activities, collective courses, personal trainers and other innovations, in line with people's consumption, living habits and needs, to be properly guided, supplemented by professional means and quality service is the fitness club long-term operation of the fundamental. Therefore, health clubs must absorb outstanding professionals, such as club theme designers, fitness class designers, star coaches, etc. These are the basic conditions for effective marketing.

In the experience of the design of the theme, in particular, should focus on fitness consumers involved in the design of 
the experience. Specifically, when designing fitness experience clubs, health clubs should make every effort to create conditions and give full play to the subjective initiative of fitness consumers so that they can participate in the dynamic design process of construction, adjustment, reconstruction and readjustment, Their related comments and suggestions for improvement can get enough attention, so that the experience of thematic design more in line with the actual situation of consumers and preferences. On the one hand, the health club should focus on providing a variety of fitness services, and establish the main principles related to fitness services; on the other hand should strive to guide fitness consumers in health club teachers and personal trainers proposed professional advice under the guidance, according to their own characteristics of highly personalized fitness experience theme design.

\section{B. Fitness brand communication}

The realization of members' fitness experience needs to be realized through the dissemination of various media. Personnel and communication are the keys to building an emotional experience through the identification, equipment, space and project activities of a fitness club. The medium of thinking and experience is mainly communication and cobranding. Marketing, people, and brand communication are the most important things for connected experience. The socalled brand, is the name, slogan, image, logo, environment and overall design that distinguish with other similar products or enterprises, the brand is value-added intangible assets, this unique intangible assets once? Accept groups, you can create identity and trust. In essence, the brand is a derivative of the club and consumers, so the brand is a product created by both businesses and consumers. Therefore, consumers naturally become the best disseminators of the brand, and the information they give out is often the most concrete, the most realistic and the most easily accepted. The theme design and brand communication are two complementary aspects in the way of experiential marketing, and the brand is the ultimate embodiment of the theme design effect. The theme design and brand communication are both accomplished by the club and consumers, both subjective and objective.

\section{Fitness club mobile experience marketing strategy: to increase social elite behavior demonstration interaction}

Mobile marketing strategy is to stimulate the interest of fitness consumers and change their life style and leisure time through the social elite, such as style stars, fashion icons and prominent figures in various industries. Specifically, actionbased experience requires that the club's management and marketing teams strive to create multiple avenues for consumers, multiple types of fitness experience opportunities, including lifestyle experiences, physical experiences, longterm behavioral patterns, and interpersonal interactions Other forms. Nike's "Just Do It" series of ads, often to the famous athletes action feature, the movement sublimated into an experience, is a very classic case of mobile marketing. The experience of the action goes beyond the range of sensations, emotions, and cognitions, as it presents different fitness content and lifestyles to members. More action experiences come from interpersonal interactions, and since actions are visible, members demonstrate their self-awareness and fitness values through their actions or lifestyles. Fitness club scenes designed to allow members to begin fitness exercise immediately. If instrumental fitness relies on the executive ability of the member, then the collective course is more conducive to members in the interaction in action.

\section{Fitness club related experience marketing strategy:}

enhance members' social self-esteem and self-confidence

Fitness club-related marketing strategy is to create the conditions so that fitness consumers self, others, fitness and social groups and groups, fitness and culture have a certain connection experience marketing strategy. Affiliate marketing varies from member to affiliate relatives with relative ease, at which point members can feel connected to other affiliates, and can be an extremely complex community of fitness brands in which fitness consumers view brands as social organizations Center [4], and assume the role of marketer. The profound experience that affiliate marketing offers members comes from the mutual influence of socio-cultural meanings and the concern of members on the social strata. The special feature of affiliate marketing is selecting the right reference group, enabling members to experience a distinctive social status that makes them happy to become part of the community and culture. In view of the general problem of interest-driven and loss of innocence in the modern society, the establishment of a cohesive workout group to enable individuals of different social status to find self-esteem and self-confidence in the club fitness activities, to obtain a positive emotional experience in addition to body shape changes, to maintain its long-term, irreplaceable fitness motivation.

\section{CONCLUSION}

At present, the overall quality of the overall marketing mode of Pingxiang City fitness club personal trainer backward, low quality, low fitness club service team. The fundamental characteristic of fitness activities is the body's participation and emotional experience, therefore, experiential marketing becomes a new marketing method of fitness clubs becomes inevitable. The practice of Baili Fitness Club's marketing proves that fitness experience is the result of a member encountering, experiencing or experiencing certain situations.put forward the Countermeasures in development of Fitness Club: to expand the premise of marketing propaganda, strengthen private coaches training, fitness club service quality improvement, the Pingxiang City fitness club really go professional and trusted industry.

\section{REFERENCES}

[1] $\mathrm{Hu}$ Feihu. Yichun City fitness club management present situation investigation and countermeasure research of $[\mathrm{J}]$. world of entrepreneurs 2013,08:30-31.

[2] Yu Xiong, Wang Wanchun. Research on the development status and Countermeasures of fitness clubs in the three tier cities -- Taking Yueyang as an example, $[\mathrm{J}]$. contemporary sports science and technology, 2013,26:117-119.

[3] Stone Xiaoxi. Fitness club in the city of Baotou on the problem and Countermeasure of $[\mathrm{J}]$. Yinshan Academic Journal (NATURAL SCIENCE), 2011,03:80-83.

[4] Zhan Zhonghua, Zhang Qingwu. Research on the development status of Anhui City Fitness Club -- Taking the fitness club in Hefei as the research object [J]. Journal of Tonghua Normal University, 2014,12:116-118. 